PESQUIMAT Revista de la Fac. CC. MM. de la

Universidad Nacional Mayor de San Marcos

Vol. IX No 1, pp. 31 - 38, LIMA - PERÚ. Julio 2006

\title{
COMPORTAMIENTO ASINTÓTICO DE LAECUACIÓN \\ DE ONDA CON CONDICIÓN DE FRONTERA TIPO NEUMANN, LOCALMENTE DISTRIBUIDA
}

\section{Alfonso Pérez Salvatierra ${ }^{1}$ y José Simeón Quique Broncano ${ }^{2}$}

RESUMEN.- En el sistema, con condiciones de frontera del tipo Neumann y disipación localmente distribuida,

$$
(*) \mid \begin{array}{lr}
u_{t t}-\Delta u+u+f(u)+a(x) u_{t}=0 & \text { en } \Omega \times(0,+\infty) \\
\frac{\partial u}{\partial v}=0 & \text { sobre } \Gamma \times(0,+\infty) \\
u(0)=u^{0} \in H^{1}, u_{t}(0)=u^{1} \in L^{2}(\Omega) & \text { en } \Omega
\end{array}
$$

donde $a(x) \geq a_{0}>0$ c.s. en $\omega, \omega \subseteq \Omega$ vecindad de $\Gamma=\partial \Omega$ el cual es un problema abierto, ver [6], se estudia el comportamiento asintótico.

PALABRAS CLAVE.- Decaimiento exponencial, multiplicadores, continuación única.

ABSTRACT.- In the system $\left({ }^{*}\right)$, with Neumann boundary conditions and locally distributed dissipation; where $a(x) \geq a_{0}>0$ a.e. in $\omega, \omega \subseteq \Omega$ neighborhood of $\Gamma=\partial \Omega$ which is an open problem, see [6], we study the asymptotic behavior.

KEY WORDS.- Exponential decay, multipliers, unique continuation.

\section{INTRODUCCIÓN}

En el presente trabajo se hace el estudio del sistema,

$$
(*) \mid \begin{array}{ll}
u_{t t}-\Delta u+u+f(u)+a(x) u_{t}=0 & \text { en } \Omega \times(0,+\infty) \\
\frac{\partial u}{\partial v}=0 & \text { sobre } \Gamma \times(0,+\infty) \\
u(0)=u^{0}, \quad u_{t}(0)=u^{1} & \text { en } \Omega
\end{array}
$$

Se tiene que el problema $\left({ }^{*}\right)$, está bien puesto en el espacio $H_{0}^{1}(\Omega) \times L^{2}(\Omega)$, es decir para datos iniciales $\left\{u^{0}, u^{1}\right\} \in H_{0}^{1}(\Omega) \times L^{2}(\Omega)$, existe solución única débil de $\left(^{*}\right)$ en la clase $u \in C([0,+\infty)$; $\left.H_{0}^{1}(\Omega)\right) \cap C^{1}\left([0,+\infty) ; L^{2}(\Omega)\right)$, y con las hipótesis siguientes:

\footnotetext{
1 Universidad Nacional Mayor de San Marcos. Facultad de Ciencias Matemáticas.e-mail:aperezs@unmsm.edu.pe
}

${ }^{2}$ Universidad Nacional Mayor de San Marcos. Facultad de Ciencias Matemáticas.e-mail:jquiqueb@unmsm.edu.pe 
$\Omega \subset \mathbb{R}^{n}$ es un abierto, acotado $(n \geq 1), \Gamma=\partial \Omega$ frontera de clase $C^{2}$.

$a(x) \in L_{+}^{\infty}(\Omega), a(x) \geq a_{0}>0$ c. s. en $\omega$, donde $\omega \subseteq \Omega$ es una vecindad de $\Gamma=\partial \Omega$.

$f$ es tal que $f(s) s \geq 0, \forall s \in \mathbb{R}$ y además $f$ es super lineal, es decir, $\exists \delta>0$ : $f(s) s \geq(2+\delta) F(s) ; \forall s \in \mathbb{R}$.

$f$ tiene la propiedad del crecimiento, es decir, $|f(x)-f(y)| \leq C\left[1+|x|^{p-1}+|y|^{p-1}\right]|x-y|$; $\forall \times, y \in \mathbb{R}$ y algún $C<0, p>1$ con $(n-2) p \leq n$.

$$
F(s)=\int_{0}^{s} f(z) d z \geq 0, \text { pues } f(s) s \geq 0 ; \forall s \in \mathbb{R}
$$

Para un $x_{0} \in \mathbb{R}^{n}$ fijo se tiene que $\partial \Omega=\Gamma=\bar{\Gamma}_{0} \cup \bar{\Gamma}_{1}$ donde $\Gamma_{0}=\{x \in \Gamma / m(x) \cdot v(x)>0\}$, $\Gamma_{1}=\{x \in \Gamma / m(x) \cdot v(x)<0\}$ con $m(x)=x-x_{0}, v(x)$, normal unitaria en un punto $x$.

Definimos la energía asociada al sistema por,

$$
E(t)=\frac{1}{2} \int_{\Omega}\left\{\left|u_{t}(x, t)\right|^{2}+|\nabla u(x, t)|^{2}+|u(x, t)|^{2}\right\} d x+\int_{\Omega} F(u(x, t)) d x
$$

Basado en el siguiente resultado de A. Ruiz [5], se tiene el siguiente lema,

Lema (Resultado de la continuación única)

Sean $b \in L^{\infty}(\omega \times(0, T)), w \in H^{1}(\Omega \times(0, T))$ tal que $w$ satisface,

$$
\mid \begin{array}{rrrl}
w^{n}-\Delta w+b(x, t) w=0 & \text { en } & Q=\Omega \times(0, t) \\
w=0 & \text { sobre } & \Sigma=\Gamma \times(0, T) \\
w=0 & \text { casi siempre en } & \omega \times(0, T)
\end{array}
$$

Entonces $w \equiv 0$.

Deducción de la energía formal:

Multiplicando el sistema $(*)$ por $u_{t}$ se obtiene

$$
\frac{d}{d t}\left\{\frac{1}{2}\left|u_{t}\right|^{2}+\frac{1}{2}|\nabla u|^{2}+\frac{1}{2}|u|^{2}+\int_{\Omega} F(u) d x\right\}=-\int_{\Omega} a(x)\left|u_{t}\right|^{2} d x
$$

de la hipótesis (1.2) se tiene que $\int_{\Omega} a(x)\left|u_{t}\right|^{2} d x>0$, entonces

$$
\frac{d}{d x} E(t)<0, \forall t \in(0,+\infty)
$$

esto es, la energía dada en (1.7) es decreciente en $(0,+\infty)$.

Usando la técnica de los multiplicadores se obtiene la estimativa de energía siguiente:

Para $T>0$ suficientemente grande; 


$$
E(T) \leq C\left\{\int_{0}^{T} \int_{\Omega} a(x)\left|u_{t}(x, t)\right|^{2} d x d t+\int_{0}^{T} \int_{\Omega}|u(x, t)|^{2} d x d t\right\}
$$

Usando técnicas de continuación única basado en el lema de la introducción se obtiene la estimativa

$$
\int_{0}^{T} \int_{\Omega}|u(x, t)|^{2} d x d t \leq C \int_{0}^{T} \int_{\Omega} a(x)|u(x, t)|^{2} d x d t
$$

Combinando (1.9) y (1.10) se obtiene que $\exists C>0$ tal que

$$
E(T) \leq C \int_{0}^{T} \int_{\Omega} a(x)\left|u_{t}(x, t)\right|^{2} d x d t
$$

De (1.11) y propiedad de teoría de semigrupo se obtiene el teorema central, sobre el decaimiento exponencial, es decir,

$$
\exists C, \gamma>0 \text { tal que } E(t) \leq C E(0) e^{-\gamma^{t}}
$$

\section{MÉTODOS Y RESULTADOS}

Los métodos y resultuados seguidos son

- Técnica de los multiplicadores:

Sirve para obtener la energía y otras estimativas para la energía.

- Técnicas de las desigualdades integrales:

Sirve para obtener estimados de la integral de energía como por ejemplo

$$
\int_{0}^{T} E(t) \leq C \int_{\Sigma_{0}} m, v\left[\left|u_{t}\right|^{2}+F(u)\right]+|\hat{x}|+\int_{\Omega}\left|(a(x) u) m \cdot \nabla u_{t}\right|
$$

donde $\Sigma_{0}=\Gamma_{0} \times(0, T), Q=\Omega \times(0, T) \quad y$

$$
\hat{x}=\left(\int_{\Omega}\left(u_{t}+a(x) u\right) m \cdot \nabla u+\alpha u\left(u_{t}+\frac{a(x) u}{2}\right)\right]_{0}^{T}
$$

- Técnicas de la continuación única:

Son usadas para estimar el término (1.10)

Usando propiedades de semigrupos, finalmente obtenemos el decaimiento de la energía dado por (1.12).

\section{RESULTADOS PREVIOS}

Considerando $\Omega \subset \mathbb{R}^{n}$ abierto regular de clase $C^{2}$, estudiamos la ecuación de onda no homogénea 


$$
(\bar{*}) \mid \begin{array}{ll}
\theta^{\prime \prime}-\Delta \theta=f & \text { en } Q=\Omega \times[0, T] \\
\theta(0)=\theta^{0}, \theta t(0)=\theta^{1} & \text { en } \Omega \\
\frac{\partial \theta}{\partial v}=0 & \text { sobre } \Sigma=\Gamma \times[0, T]
\end{array}
$$

\section{Lema 1}

Sea $\Omega$ un dominio acotado de $\mathbb{R}^{n}$ con frontera $\Gamma$ de clase $C^{2}, \quad q=\left(q_{k}\right)_{k \geq 1}$ un campo vectorial de clase $\left[C^{1}(\Omega)\right]^{n}$. Entonces, para toda solución débil $\theta=\theta(x, t)$ del sistema $(\bar{*})$,

$$
\text { (es decir, } \forall\left\{\theta^{0}, \theta^{1}\right\} \in H_{0}^{1}(\Omega) \times L^{2}(\Omega), f \in L^{1}\left(0, T ; L^{2}(\Omega)\right) \text { ) }
$$

se tiene

$$
\begin{aligned}
\int_{\Omega}\left(\theta^{\prime \prime}-\Delta \theta\right) q \cdot \nabla \theta & =-\frac{1}{2} \int_{\Sigma} q \cdot v\left|\theta^{\prime}\right|^{2}+\left(\int_{\Omega} \theta^{\prime} q \cdot \nabla \theta\right]_{0}^{T}+ \\
& +\frac{1}{2} \int_{Q} \operatorname{div} \cdot(q)\left[\left|\theta^{t}\right|^{2}-|\nabla \theta|^{2}\right]+\int_{Q} \frac{\partial\left(q_{k}\right)}{\partial x_{j}} \frac{\partial \theta}{\partial x_{k}} \frac{\partial \theta}{\partial x_{j}}
\end{aligned}
$$

\section{Demostración.-}

Usamos la técnica de los multiplicadores. Multiplicando $(\bar{*})$ por $q_{k} \frac{\partial \theta}{\partial x_{k}}$, donde $\theta$ es la solución débil del sistema $(\bar{*})$ se obtiene el lema, ver [4].

\section{Lema 2}

Para $u$ solución débil de (*) y con las hipótesis dados en (1.1) y (1.2) se tiene

$$
\begin{aligned}
\frac{1}{2} \int_{Q} \operatorname{div} \cdot(q) & {\left[\left|\theta_{t}\right|^{2}-|\nabla \theta|^{2}-2|u|^{2}-2 F(u)\right]+\int_{Q} q \cdot \nabla u\left[a(x) u_{t}-u\right]+} \\
& +\int_{Q} \frac{\partial u}{\partial x_{j}} \frac{\partial u}{\partial x_{k}} \frac{\partial}{\partial x_{j}}\left(q_{k}\right)=\frac{1}{2} \int_{\Sigma} q \cdot v\left[\left|\theta_{t}\right|^{2}-2|u|^{2}-2 F(u)\right]-\left(\int_{\Omega} u_{t} q \cdot \nabla u\right]_{0}^{T}
\end{aligned}
$$

\section{Demostración}

Se multiplica $\left(^{*}\right)$ por $q . \nabla u$ y se aplica la técnica de los multiplicadores.

Observación.- El lema precedente es también válido para $q \in\left(W^{1, \infty}(\Omega)\right)^{n}$.

\section{Lema 3}

Con las hipótesis dadas en (1.1), (1.2) y (1.6) se tiene 


$$
\begin{array}{r}
\left(\int_{\Omega} m \cdot \nabla u_{t}+a(x) u\right]_{0}^{T}+\frac{n}{2} \int_{Q}\left|u_{t}\right|^{2}+\left(1-\frac{n}{2}\right) \int_{Q}|\nabla u|^{2}- \\
-n+\int_{Q}|u|^{2}+n \int_{Q} F(u)-\int_{Q} a(x) u m \cdot \nabla u_{t}-\int_{Q} u m \cdot \nabla u \leq \\
\leq \int_{\Sigma_{0}} m \cdot v\left[\left|u_{t}\right|^{2}-|u|^{2}+F(u)\right]
\end{array}
$$

\section{Lema 4}

Si $\Omega \subset \mathbb{R}^{n}$ es un abierto regular y $u$ solución débil de $\left(^{*}\right)$, entonces se obtiene

$$
\left(\int_{\Omega} u\left(u_{t}+\frac{a(x) u}{2}\right)\right]_{0}^{T}=\int_{Q}\left[\left|u_{t}\right|^{2}-|\nabla u|^{2}\right]-\int_{Q}\left[u f(u)+|u|^{2}\right]
$$

\section{Demostración}

Basta multiplicar por $\xi u$ al sistema $\left({ }^{*}\right) \operatorname{con} \xi \in W^{1, \infty}(\Omega)$ y luego, tomar $\xi=1$.

\section{Lema 5}

Con las hipótesis de (1.1) - (1.6), se tiene que $\exists C>0$ tal que

$$
C \int_{0}^{T} E(t) \leq \int_{\Sigma_{0}} m \cdot v\left|\left[\left|u_{t}\right|^{2}+|u|^{2}+F(x)\right]\right|+|\hat{x}|+\int_{Q}\left|a(x) u m \cdot \nabla u_{t}\right|
$$

donde $\hat{x}=\left(\int_{\Omega}\left\{m \cdot \nabla u\left[u_{t}+a(x) u\right]+\alpha u\left(u_{t}+\frac{a(x) u}{2}\right)\right\}\right]_{0}^{T}, \alpha>0$

\section{Demostración}

Combinando los lemas (3) y (4) se obtiene el lema precedente.

\section{Lema 6}

Con las hipótesis de (1.1) - (1.6) se obtiene

$$
\left(\int_{\Omega} u\left(u_{t}+\frac{a(x) u}{2}\right)\right]_{0}^{T}=\int_{Q}\left[\left|u_{t}\right|^{2}-|\nabla u|^{2}\right]-\int_{Q}|u|-\int_{Q} u f(u)
$$

\section{Demostración}

Multiplicando por $\xi(x)$ u $a(x)$ al sistema $\left({ }^{*}\right)$ e integrando de 0 a T, se obtiene el resultado propuesto. 


\section{Proposición 1}

Con las hipótesis de (1.1) - (1.6) se obtiene,

$$
\begin{gathered}
T E(T) \leq C_{1}\left\{\int_{Q} a(x)\left|u_{t}\right|^{2}+\int_{Q}|u|^{2}+\left|\left(\int_{\Omega} u_{t} h . \nabla u\right]_{0}^{T}\right|+|\hat{x}|+|\hat{y}|\right\} \\
\operatorname{con} \hat{y}=\left(\int_{\Omega} \eta\left(u_{t}+\frac{a(x) u}{2}\right)\right]_{0}^{T}
\end{gathered}
$$

\section{Demostración}

Resulta de los lemas 5 y 6.

Desde que la energía es decreciente, se tiene:

$$
T E(T) \leq \int_{0}^{T} E(t) d t
$$

\section{Lema 7}

Con la hipótesis de (1.1) - (1.4) se tiene:

$$
|\hat{x}|+|\hat{y}|+\left|\left(\int_{\Omega} u_{t} h . \nabla u\right]_{0}^{T}\right| \leq C_{2}\left(2 E(T)+\int_{Q} a(x)\left|u_{t}\right|^{2}\right)
$$

\section{Proposición 2}

Con las hipótesis de (1.1) - (1.6) obtenemos,

$$
E(T) \leq C\left(\int_{Q} a(x)\left|u_{t}\right|^{2}+\int_{Q}|u|^{2}\right), C>0 .
$$

\section{Demostración}

Del lema 7 en la proposición 1:

$$
\begin{aligned}
T E(T) & \leq C_{1}\left\{\int_{\underline{b}} a(x)\left|u_{t}\right|^{2}+\int_{\underline{b}}|u|^{2}\right\}+C_{1}\left\{\left|\left(\int_{\Omega} u_{1} h \cdot \nabla u\right]_{0}^{T}\right|+|\hat{x}|+|\hat{y}|\right\} \\
& \leq C_{1}\left\{\int_{\underline{Q}} a(x)\left|u_{t}\right|^{2}+\int_{\underline{O}}|u|^{2}\right\}+C_{1} C_{2}\left(2 E(T)+\int_{\underline{Q}} a(x)\left|u_{t}\right|^{2}\right) \\
& \leq C_{3}\left\{\int_{\underline{b}} a(x)\left|u_{t}\right|^{2}+\int_{\underline{b}}|u|^{2}+2 E(T)+\int_{\underline{Q}} a(x)\left|u_{t}\right|^{2}\right\}, C_{3}=\max \left\{C_{1}, C_{1} C_{2}\right\} \\
\left(T-2 C_{3}\right) E(T) \leq K\left\{\int_{\underline{b}} a(x)\left|u_{t}\right|^{2}+\int_{\underline{Q}}|u|^{2}\right\}, K=\max \left\{2 C_{3}, C_{3}\right\} & \\
E(T) & \leq \frac{K}{T-2 C_{3}}\left\{\int_{\underline{Q}} a(x)\left|u_{t}\right|^{2}+|u|^{2}\right\}
\end{aligned}
$$

Para T suficientemente grande tal que $T-2 C,>0$, obtenemos la proposición 2 . 


\section{Proposición 3}

Con las hipótesis de (1.1) - (1.4) obtenemos,

$$
\int_{Q}|u|^{2} \leq C \int_{Q} a(x)|u|^{2}
$$

\section{Demostración}

La demostración es por el absurdo, usando el principio de la continuación única que aparece en A. Ruiz [5], construimos un sistema estacionario cuya solución lleva a una contradicción.

\section{EL TEOREMA CENTRAL}

Mediante un teorema proponemos nuestro resultado principal acerca del decaimiento exponencial de la energía para soluciones débiles del sistema $\left({ }^{*}\right)$.

\section{Teorema}

Si $\Omega \subset \mathbb{R}^{n}$ es un abierto acotado con frontera de clase $C^{2}$, y se satisfaen las condiciones (1.1) (1.6); entonces existen constantes $C, \gamma>0$ tal que,

$$
E(t) \leq C E(0) e^{-\gamma t}, \forall t \geq 0
$$

para toda solución débil de $\left(^{*}\right)$.

\section{Demostración}

De la proposición 3, en la proposición 2 se tiene que, $\exists C_{0}>0$ tal que

$$
E(T) \leq C_{0} \int_{0}^{T} \int_{\Omega} a(x)\left|u_{t}\right|^{2} d x d t
$$

desde que,

$$
\frac{d}{d t} E(t)=-\int_{\Omega} a(x)\left|u_{t}\right|^{2} d x
$$

integrando de 0 a T; obtenemos

$$
E(T)-E(0)=-\int_{0}^{T} \int_{\Omega} a(x)\left|u_{t}\right|^{2} d x d t
$$

Combinando (4.1) y (4.2) se obtiene

$$
E(T) \leq \frac{C_{0}}{1+C_{0}} E(0), C=\frac{C_{0}}{1+C_{0}}<1, T>0 .
$$

Luego, por propiedad de teoría de semigrupos y como el problema $\left({ }^{*}\right)$ está bien puesto, se obtiene que

$$
E(t) \leq K E(0) e^{-\gamma t}, \forall t \geq 0
$$


para toda solución débil de $(*)$, donde

$$
C=\frac{C_{0}}{1+C_{0}}, \quad \gamma=\frac{1}{T} \log C ; \quad K=\frac{C_{0}}{1+C_{0}}
$$

\section{COMENTARIOS}

Existen muchos métodos para el estudio del decaimiento de la energía con condiciones iniciales y de frontera; al respecto puede verse [1], [2] y [3]. En este trabajo se usó el método de la continuación única combinado con el método de los multiplicadores e integrales en la frontera.

El presente articulo es uno de los muchos trabajos realizados recientemente, que consiste en realizar perturbaciones físicas en una vecindad de la frontera, para conocer el efecto en todo su dominio, ver [7], [8], [9], [10] y [11].

\section{CONCLUSIONES}

Se observa que el método usado para obtener el decaimiento exponencial del sistema $(*)$, el cual ha usado el principio de la continuación única, según A. Ruiz [5], puede también adaptarse a otros sistemas a los cuales se les puede agregar o variar datos en la frontera. Además es posible aplicar este método a otros modelos, comos son: Von Karman con condiciones de disipación en la frontera, placas con condiciones mixtas en la frontera de tipo Dirichlet - Newmann, etc, que podrían ser materia de otros estudios.

El método usado, es una de las múltiples maneras de obtener el decaimiento exponencial de la energía de ciertos sistemas.

\section{REFERENCIAS BIBLIOGRÁFICAS}

[1] Haraux A. Stabilization of trayectories for some weakly damped hyperbolic equations, J. Differential equation 59, 145 - 154 (1985).

[2] Komornik V. Exact controllability in short time for the wave equation, Ann. Inst. H. Poincaré, Analyse Nonlinéaire 6, 153 - 164 (1989).

[3] Komornik V. and Zuazua E. Stabilizatión frontiére de L'équation des ondes: Une Méthode directe, C. R. Acad. Sci. Paris 305, 605 - 608 (1987).

[4] Lions J. L. Contrôlabilité exacte, perturbations et stabilizations de systémes Distribués. Tome 1. Contrôlabilité Exacte, RMA 8, Masson, (1988).

[5] Ruiz A. Unique continuation for weak solution of the wave equation plus a potencial Journal, Math pure Applicada 71, 455 - 467 (1992).

[6] Zuazua E. Exponential decay for the semilinear wave equation with locally distributed damping. Comm PDE, 15, pp 205 - 235 (1990).

[7] Muñoz J., Bisognin V. and Bisognin E. Exponential decay to partially thermoelastic materials, (1996).

[8] Pérez A. Decaimento de soluçõoes de equações parcialmente Viscoelásticas. Tese de Doutorado. UFRJ. Brasil (1997).

[9] Pérez A. Decaimento exponencial da soluçâo da onda com potencial e amortecimento localmente distribuido. UNMSM. Revista Pesquimat. Lima - Perú (2004).

[10] Cabanillas E. Estabilización de la energía para una ecuación de Kirchoff con disipación localizada, Proy. Invest. Inst. Invest. UNMSM. Lima - Perú. (2004).

[11] Portillo H. and Muñoz J. Sobre um problema de contato unidimensional de Ondas elásticas localmente amortecidas. SBA $46^{\circ}$ Seminario Brasileiro de Análise. Brasil (1997). 